\title{
THERE ARE MANY OSTASZEWSKI SPACES
}

\author{
TIMOTHY J. LABERGE
}

(Communicated by Franklin D. Tall)

\begin{abstract}
We show that $\diamond \omega_{1}$ implies there is a family of $2^{\omega_{1}}$ many pairwise nonhomeomorphic Ostaszewski spaces, with the additional property that any continuous image of one in another is compact. We also present diamond versions of Fleissner's forcing results concerning the normality of products of Ostaszewski spaces and their compactifications.
\end{abstract}

\section{INTRODUCTION}

By an Ostaszewski space, we mean a topological space whose point set is the ordinal $\omega_{1}$ that is first countable, locally countable, countably compact, hereditarily separable, perfectly normal, zero-dimensional, locally compact, and not Lindelöf. It is folklore that either forcing or the combinatorial principle $\diamond \omega_{1}$ can be used to construct nonhomeomorphic Ostaszewski spaces. We use $\diamond \omega_{1}$ to construct a family of $2^{\omega_{1}}$ many Ostaszewski spaces with the stronger property that the continuous image of one in another is always compact. Since an Ostaszewski space is a first countable, locally countable topological space on the ordinal $\omega_{1}$, this is the largest possible size for such a family.

In [F] Fleissner has obtained results concerning the normality of products of Ostaszewski spaces and their compactifications by adding $\omega_{1}$ many Cohen reals to a model of $\mathrm{CH}$. We give versions of these results that use $\nabla_{\omega_{1}}$ instead of forcing.

Notation. We establish some notation that will be used throughout. Let $X$ be an Ostaszewski space.

(1) $\nabla_{\omega_{1}}$ is the assertation that there is a family $\left\{E_{\alpha}: \alpha \in \omega_{1}\right\}$ such that each $E_{\alpha} \subseteq \alpha$ and for each $A \in\left[\omega_{1}\right]^{\omega_{1}}$, the set $S=\left\{\alpha \in \omega_{1}: A \cap \alpha=E_{\alpha}\right\}$ is stationary.

(2) $\left\{\lambda_{\alpha}: \alpha \in \omega_{1}\right\}$ is the increasing 1-1 enumeration of the limit ordinals in $\omega_{1}$.

(3) $\$$ is the assertation that there is a family $\left\{c\left(\lambda_{\alpha}\right): \alpha \in \omega_{1}\right\}$ such that each $c\left(\lambda_{\alpha}\right)$ is an $\omega$ sequence of ordinals cofinal in $\lambda_{\alpha}$ and each uncountable subset of $\omega_{1}$ contains some $c\left(\lambda_{\alpha}\right)$.

(4) $T=\left(<\omega_{1} 2, \subset\right)$.

Received by the editors November 20, 1991.

1991 Mathematics Subject Classification. Primary 54A10; Secondary 54A35, 54B99, 54G15.

Key words and phrases. Diamond, club, Ostaszewski, normality of products. 
(5) For $\alpha \in \omega_{1}$ and $t \in \operatorname{Lev}_{\alpha} T$, both $X_{\alpha}$ and $X(t)$ denote topological spaces with point set $\lambda_{\alpha}$.

(6) If $t \in \operatorname{Lev}_{\alpha}(T)$, then $t^{-}=t \cup\{(\alpha, 0)\}$ and $t^{+}=t \cup\{(\alpha, 1)\}$.

(7) $\Delta=\{(x, x): x \in X\}$.

(8) Suppose $f: X \rightarrow X$. Then $\Gamma(f)=\{(x, f(x)): x \in X\}$. Thus, if $f$ is the identity on $X$ then $\Delta=\Gamma(f)$.

The basic construction. Our constructions generalize Ostaszewski's construction in [O], which we will refer to as the basic construction. We assume $\mathrm{CH}$ and the combinatorial principle $\$$; this combination is equivalent to $\nabla_{\omega_{1}}$ (see [D]). By CH, we can find an onto map $F: \omega_{1} \backslash \omega \rightarrow\left[\omega_{1}\right]^{\omega}$ such that $F(\alpha) \subseteq \alpha$, for all $\alpha \in \omega_{1}$. Let $\left\{c\left(\lambda_{\alpha}\right): \alpha \in \omega_{1}\right\}$ be a \& sequence.

We proceed by induction on $\alpha \in \omega_{1}$. At stage $\alpha$, we construct a topology $\tau(\alpha)$ on the ordinal $\lambda_{\alpha}$ to obtain a space $X_{\alpha}$. The induction also satisfies the following: whenever $\beta<\alpha \leq \omega_{1}$,

(1) $X_{\beta}$ is an open subspace of $X_{\alpha}$.

(2) $X_{\alpha}$ is zero-dimensional, locally compact, and metrizable.

(3) If $\lambda_{\beta} \leq \mu<\lambda_{\alpha}$, then $\mu$ is in the $X_{\alpha}$-closure of $c\left(\lambda_{\beta}\right)$.

(4) If $\alpha=\nu+1$, then $F(\nu)$ has an accumulation point in $X_{\alpha}$

We begin by taking $\tau(0)$ to be the discrete topology on $\lambda_{0}=\omega$. This clearly satisfies (1)-(4). If $\alpha$ is a limit, we take $\tau(\alpha)$ to be the topology on $\lambda_{\alpha}$ generated by the union of the $\tau(\beta)$, for $\beta<\alpha$ (i.e., we take the direct limit). This also satisfies (1)-(4). Thus, the only troublesome case is when $\alpha=\nu+1$. We will use the following lemma, which is also due to Ostaszewski.

Lemma 0. Let $X$ be a countable, zero-dimensional, locally compact, metrizable space with a compatible metric. Let $A_{1}, \ldots, A_{n}$ be closed discrete subsets of $X$. Then there is a space $Y$ that contains $X$ as an open subspace, such that $Y$ is also countable, zero-dimensional, locally compact, and metrizable, with $Y \backslash X$ a closed discrete subset of $Y$, and such that each point of $Y \backslash X$ is an accumulation point of each $A_{i}$.

Proof. A complete proof can be found in [O]; we will be content to sketch the necessary construction. For concreteness, we will assume that $n=2$, so set $A=$ $A_{1}$ and $B=A_{2}$. Some of the applications will require a specific enumeration of $A$ and $B$, so suppose we have $A=\left\{a_{n}: n \in \omega\right\}$ and $B=\left\{b_{n}: n \in \omega\right\}$.

We would like to assume that $A$ and $B$ are either disjoint or equal. We can ensure this by thinning $A$ and $B$, but some of our product constructions require that if we thin $A$ to $\left\{a_{n_{k}}: k \in \omega\right\}$ then we thin $B$ to $\left\{b_{n_{k}}: k \in \omega\right\}$. Having done this, reindex so that $A=\left\{a_{n}: n \in \omega\right\}$ and $B=\left\{b_{n}: n \in \omega\right\}$.

By induction on $n \in \omega$, find neighborhoods $U_{n}$ of $a_{n}$ and $V_{n}$ of $b_{n}$ such that $U_{n}$ and $V_{n}$ are disjoint, clopen-compact sets of diameter at most $1 / n$, and such that $\left\{U_{n}: n \in \omega\right\} \cup\left\{V_{n}: n \in \omega\right\}$ is a discrete family of subsets of $X$.

Let $\left\{y_{n}: n \in \omega\right\}$ be countably many points not in $X$. Partition $\omega$ into infinitely many disjoint infinite sets $\left\{W_{n}: n \in \omega\right\}$. Set $Y=X \cup\left\{y_{n}: n \in \omega\right\}$. Topologize $Y$ so that points of $X$ retain their $X$-neighborhood bases and so that a base at $y_{n}$ is a set of the form $B\left(y_{n}, k\right)=\left\{y_{n}\right\} \cup \bigcup_{m \in W_{n}, m \geq k} U_{m} \cup V_{m}$. Then the space $Y$ is as required. 
Returning to the basic construction, we find closed discrete subsets $A$ and $B$ of $X_{\nu}$ as follows: set $A=c\left(\lambda_{\nu}\right)$. If $F(\nu)$ is a closed discrete of $X_{\nu}$, set $B=F(\nu)$; otherwise, set $B=A$. Using Lemma 0 , find an extension $Y=X_{\nu} \cup\left\{y_{n}: n \in \omega\right\}$ of $X_{\nu}$. Take $\tau(\alpha)$ to be the topology on $\lambda_{\alpha}$ obtained by identifying $y_{n}$ with the ordinal $\lambda_{\nu}+n$. This completes the successor stage of the induction.

The construction continues until $\alpha=\omega_{1}$. Let $X$ be the direct limit of $\left\{X_{\alpha}: \alpha \in \omega_{1}\right\}$. Then $X$ is an Ostaszewski space.

Remark. Having given the basic construction, in our applications we will often just give the necessary modifications to the successor stage and/or Lemma 0.

\section{A FAMILY OF $2^{\omega_{1}}$ MANY DisTinCt OstaszewSKi SPACES}

As already noted, it is well known that there are nonhomeomorphic Ostaszewski spaces. We will construct a family of $2^{\omega_{1}}$ many Ostaszewski spaces with the stronger property that the continuous image of one in another is always compact. Ostaszewski spaces are locally countable and first countable, so this is the largest possible size for such a family.

We describe the combinatorics that our construction will use. We assume the combinatorial principle diamond [K]. Let $\left\{\left(f_{\alpha}, s_{\alpha}, t_{\alpha}\right): \alpha \in \omega_{1}\right\}$ be a diamond sequence such that for each $(f, b, d) \in{ }^{\omega_{1}} \omega_{1} \times{ }^{\omega_{1}} 2 \times{ }^{\omega_{1}} 2$, there are uncountably many $\alpha \in \omega_{1}$ such that

$$
\left(f_{\alpha}, s_{\alpha}, t_{\alpha}\right)=\left(f \left\lceil\lambda_{\alpha}, b\lceil\alpha, d\lceil\alpha)\right.\right.
$$

Such a sequence can be obtained from the usual diamond sequence on $\omega_{1}$ by standard coding arguments and the fact that on a club, $\lambda_{\alpha}=\alpha$. Our plan is to interpret $\left(f_{\alpha}, s_{\alpha}, t_{\alpha}\right)$ as ' $f_{\alpha}$ is a partial function from a space $X(b)$ to a space $X(d)$ ' and to destroy the continuity of $f_{\alpha}$ at stage $\alpha+1$, if necessary.

We now describe the inductive construction of the spaces. For each $s \in T$, we will construct a space $X(s)=\left(\lambda_{\alpha}, \tau(s)\right)$, where $\alpha$ is the height of $s$ in $T$. The induction, which is on $\alpha \in \omega_{1}$, constructs all $\tau(s), s \in \operatorname{Lev}_{\alpha} T$, at stage $\alpha$, and also satisfies, whenever $r \subset s \in \operatorname{Lev}_{\alpha} T$,

(1) $X(r)$ is an open subspace of $X(s)$.

(2) $X(s)$ is zero-dimensional, locally compact, and metrizable.

(3) If $\operatorname{ht}(r)=\alpha^{\prime}, \operatorname{ht}(s)=\alpha$, and $\lambda_{\alpha^{\prime}} \leq \mu<\lambda_{\alpha}$, then $\mu$ is in the $X(s)$-closure of $c\left(\lambda_{\alpha^{\prime}}\right)$.

(4) If $\alpha=\nu+1$, then $F(\nu)$ has an accumulation point in $X(s)$.

(5) If $\alpha=\nu+1, s_{\nu} \neq t_{\nu}$, and for some $\rho \leq \nu$, range $\left(f_{\nu}\right) \supseteq c\left(\lambda_{\rho}\right)$, then $f_{\nu}$ has no continuous extension as a map from the space $X\left(s_{\nu} \cup(\nu, \epsilon)\right)$ to the space $X\left(t_{\nu} \cup(\nu, \delta)\right)$, for each $\epsilon, \delta \in\{0,1\}$.

After completing the induction we will define, for each $b \in \omega^{\omega_{1}} 2$, a topology $\tau(b)$ on $\omega_{1}$ by taking the direct limit of the $X(s)$ such that $s \in T$ and $s \subset b$. Set $X(b)=\left(\omega_{1}, \tau(b)\right)$, then our family of Ostaszewski spaces is $\{X(b): b \in$ $\left.\omega_{1} 2\right\}$.

To begin the induction, take $\tau(\varnothing)$ to be the discrete topology on $\lambda_{0}$. Since $\lambda_{0}=\omega,(1)-(5)$ are clearly satisfied. When $\alpha$ is a limit and $s \in \operatorname{Lev}_{\alpha} T$, we take $\tau(s)$ to be the direct limit of the $\tau(r)$, such that $r \subset s$. This also satisfies (1)-(5). Thus, the only troublesome case is when $\alpha=\nu+1$.

To complete the induction when $\alpha=\nu+1$, we must construct $\tau(s)$ for each 
$s \in \operatorname{Lev}_{\alpha} T$. For $s \notin\left\{s_{\nu}^{+}, s_{\nu}^{-}\right\}$, construct $X(s)$ from $X(s \mid \nu)$ exactly as in the basic construction. For brevity, we will only construct $X\left(s_{\nu}^{-}\right) ; X\left(s_{\nu}^{+}\right)$is constructed analogously.

We begin by finding closed discrete subsets $A, B, C, D, E$, and $F$ of $X\left(s_{\nu}\right)$. Find $A$ and $B$ as in the basic construction. Now consider $f_{\nu}$ as a function from $X\left(s_{\nu}\right)$ into $X\left(t_{\nu}^{-}\right)$. If $s_{\nu} \neq t_{\nu}, f_{\nu}$ is continuous, and the range of $f_{\nu}$ contains a $c\left(\lambda_{\rho}\right)$, for some $\rho \leq \nu$, take ordinals $\mu$ and $\mu^{\prime}$ with $\lambda_{\nu} \leq \mu<\mu^{\prime}<\lambda_{\alpha}$. Note that by (3) of the induction hypothesis, in $X\left(t_{\nu}^{-}\right)$ both $\mu$ and $\mu^{\prime}$ are in the closure of the countable set $c\left(\lambda_{\rho}\right)$. Since $X\left(t_{\nu}^{-}\right)$is a zero-dimensional, locally compact, metrizable space, there are disjoint, clopencompact neighborhoods $U$ and $U^{\prime}$ of $\mu$ and $\mu^{\prime}$, respectively. Metrizable spaces are Frechet-Urysohn, so there are sequences $\left(x_{n}\right)_{n \in \omega} \subseteq U \cap c\left(\lambda_{\rho}\right)$ and $\left(y_{n}\right)_{n \in \omega} \subseteq U^{\prime} \cap c\left(\lambda_{\rho}\right)$ that converge to $\mu$ and $\mu^{\prime}$, respectively.

Now, sup $\left(\operatorname{range}\left(f_{\nu}\right)\right) \leq \lambda_{\nu}$, and $\lambda_{\nu} \leq \mu<\mu^{\prime}<\lambda_{\nu+1}$, so that $\left\{f_{\nu}^{-1}\left(x_{n}\right)\right.$ : $n \in \omega\}$ and $\left\{f_{\nu}^{-1}\left(y_{n}\right): n \in \omega\right\}$ are discrete families of closed sets in $X\left(s_{\nu}\right)$. Taking $c_{n} \in f_{\nu}^{-1}\left(x_{n}\right)$ and $d_{n} \in f_{\nu}^{-1}\left(y_{n}\right)$, we obtain countable, closed discrete sets $C=\left\{c_{n}: n \in \omega\right\}$ and $D=\left\{d_{n}: n \in \omega\right\}$. If the above requirements are not met, we set $C=D=A$. In a similar manner, consider $f_{\nu}$ as a function from $X\left(s_{\nu}\right)$ into $X\left(t_{\nu}^{+}\right)$and obtain closed discrete sets $E$ and $F$.

We are now ready to define $\tau\left(s_{\nu}^{-}\right)$. Using Lemma 0 , we find an extension $Y$ of $X\left(s_{\nu}\right)$ such that each $y \in Y \backslash X\left(s_{\nu}\right)$ is an accumulation point of $A, B, C$, $D, E$, and $F$. We identify $Y \backslash X\left(s_{\nu}\right)$ with the countable set $\lambda_{\alpha} \backslash \lambda_{\nu}$ and thus obtain the topology $\tau\left(s_{\nu}^{-}\right)$on $\lambda_{\alpha}$.

Clearly (1)-(4) of the induction hypothesis are satisfied. To check (5), recall that if $f: W \rightarrow Z$ is continuous, and $H \subseteq W$, then $f\left[\operatorname{cl}_{W} H\right] \subseteq \operatorname{cl}_{Z} f[H]$. Suppose $s_{\nu} \neq t_{\nu}, f_{\nu}$ is a continuous function from $X\left(s_{\nu}\right)$ to $X\left(t_{\nu}\right)$, the range of $f_{\nu}$ contains some $c\left(\lambda_{\rho}\right)$, where $\rho \leq \nu$, and that we can extend $f_{\nu}$ to a continuous function $f$ mapping $X=X\left(s_{\nu}^{-}\right)$to $Y=X\left(t_{\nu}^{-}\right)$. (If this is not the case, then hypothesis (5) holds vacuously.) By construction, there is an $x \in$ $\left(\mathrm{cl}_{X} C \cap \mathrm{cl}_{X} D\right) \backslash \lambda_{\nu}$. By the continuity of $f$,

$$
f(x) \in \operatorname{cl}_{Y} f[C] \cap \operatorname{cl}_{Y} f[D] \subseteq \operatorname{cl}_{Y} U \cap \operatorname{cl}_{Y} U^{\prime}=\varnothing,
$$

a contradiction. Similary, using the sets $E$ and $F$, we reach a contradiction if we assume $f_{\nu}$ extends to a continuous function from $X\left(s_{\nu}^{-}\right)$to $X\left(t_{\nu}^{+}\right)$. This completes the induction when $\alpha=\nu+1$.

Having define our family of Ostaszewski spaces, we need to show that if we have a continuous function $f: X(b) \rightarrow X(d)$ and the range of $f$ is noncompact, then $b=d$. Suppose $H=f[X(b)]$ is not compact. Since $X(b)$ is normal and almost compact (see [FKL]), we have that $H$ is uncountable and so contains some $c\left(\lambda_{\rho}\right)$. By diamond, there is a $\nu \in \omega_{1}$ so that $\left(f \mid \lambda_{\nu}, b \backslash \nu\right.$, $d\lceil\nu)=\left(f_{\nu}, s_{\nu}, t_{\nu}\right)$. Recalling the inductive construction of $X(b)$, we see that unless $b=d, f\left\lceil\lambda_{\nu}=f_{\lambda_{\nu}}\right.$ cannot be extended to a continuous function from $X(b\lceil(\nu+1))$ into $X(d\lceil(\nu+1))$, so the proof is complete.

\section{NORMALITY OF PRODUCTS}

In this section, we give diamond versions of Fleissner's recent forcing results (see [F]) concerning the normality of products of Ostaszewski spaces and their compactifications. The essential new concepts are contained in the following definitions, which are derived from the notion of a 2-uncountable set (see [B]). 
Definition. Let $X$ and $Y$ be topological spaces.

(1) A subset of $A \subseteq X \times Y$ is skeletal if it is of the form $S\left(K_{0}, K_{1}\right)=$ $\left(K_{0} \times Y\right) \cup\left(X \times K_{1}\right)$, where $K_{0}$ is a compact subset of $X$ and $K_{1}$ is a compact subset of $Y$.

(2) A subset of $X \times Y$ is large if it is not contained in any skeletal set.

(3) A subset $A \subseteq X \times Y$ is wispy if it is closed and noncompact, but for each $x \in X$, the section $\{y \in Y:(x, y) \in A\}$ is compact, and for each $y \in Y$, the section $\{x \in X:(x, y) \in A\}$ is compact. (Note that wispy implies large.)

The following lemma is from $[\mathrm{F}]$ and is proved in detail there. The essential element of the proof is the use of Glicksburg's theorem to show that if $\mathrm{X}$ and $Y$ are Ostaszewski spaces, then $\beta(X \times Y)=\beta X \times \beta Y$.

Lemma 1. Let $X$ and $Y$ be Ostaszewski spaces.

(1) The product $X \times Y$ is nonnormal if and only if there is a pair of disjoint wispy sets in $X \times Y$.

(2) The products $\beta X \times Y$ and $X \times \beta Y$ are both normal if and only if there are no wispy sets in $X \times Y$.

Corollary 2. Let $X$ be an Ostaszewski space.

(1) The product $X \times X$ is nonnormal if and only if there is a wispy set disjoint from $\Delta$ in $X \times X$.

(2) If $X$ has an autohomeomorphism with no fixed points, then $X \times X$ is not normal.

(3) The product $X \times \beta X$ is never normal.

(4) If $\beta X \times Y$ and $X \times \beta Y$ are both normal and $f: X \rightarrow Y$, then the range of $f$ is a compact subset of $Y$.

Proof. Note that $\Delta$ is a wispy subset of $X \times X$.

(1) Easy.

(2) If $\sigma$ is such an autohomeomorphism, then $\Gamma(\sigma)$ is a wispy set disjoint from $\Delta$.

(3) Easy, but also a consequence of Tamano's theorem [P].

(4) If the range of $f$ is noncompact, then $\Gamma(f)$ is wispy.

Remark. Concerning the above corollary, we can also construct a family of $2^{\omega_{1}}$ many Ostaszewski spaces with the property that $\beta X \times Y$ is always normal. Note that by (4) of the above lemma, this is stronger than requiring all continuous images of one space in another to be compact. It is also possible to construct such families with the property that each space in the family has normal (or nonnormal) square. To construct such families, proceed as before, but replace the basic construction of an Ostaszewski space with one of the constructions described below, taking care to kill counterexamples as necessary. The details are left to the interested reader.

$X \times X$ nonnormal. We first construct an Ostaszewski space $\mathrm{X}$ such that $X \times X$ is nonnormal. This construction is well known; we include it for completeness. By Corollary 2 , it suffices to construct $X$ so that the permutation $\sigma$ on $\omega_{1}$ defined by

$$
\sigma\left(\lambda_{\alpha}+2 n\right)=\lambda_{\alpha}+2 n+1, \quad \sigma\left(\lambda_{\alpha}+2 n+1\right)=\lambda_{\alpha}+2 n
$$

is an autohomeomorphism. 
We only need to modify the basic construction at stages such that $\alpha=\nu+1$; it suffices to make $\sigma \mid \lambda_{\alpha}$ a homeomorphism. We first find the closed discrete sets $A$ and $B$ as in the basic construction. By thinning, we can assume that $A, \sigma[A], B$, and $\sigma[B]$ are pairwise disjoint. List $A=\left\{a_{n}: n \in \omega\right\}$ and $B=$ $\left\{b_{n}: n \in \omega\right\}$. By induction on $n \in \omega$, choose clopen-compact neighborhoods $U_{n}$ of $a_{n}$ and $V_{n}$ of $b_{n}$ of diameter at most $1 / n$, with the additional requirement that the family

$$
\left\{A_{n}: n \in \omega\right\} \cup\left\{\sigma\left[A_{n}\right]: n \in \omega\right\} \cup\left\{B_{n}: n \in \omega\right\} \cup\left\{\sigma\left[B_{n}\right]: n \in \omega\right\}
$$

is a discrete family of subsets of $X_{\nu}$.

We define neighborhoods in a manner similar to Lemma 0: partition $\omega$ into infinitely many disjoint infinite sets $\left\{W_{n}: n \in \omega\right\}$. For each $k \in \omega$, define a neighborhood $B\left(\lambda_{\alpha}+2 n, k\right)$ of $\lambda_{\alpha}+2 n$ by

$$
B\left(\lambda_{\alpha}+2 n, k\right)=\left\{\lambda_{\alpha}+2 n\right\} \cup \bigcup_{\substack{m \in W_{n} \\ m \geq k}} U_{2 m} \cup \sigma\left[U_{2 m+1}\right] \cup V_{2 m} \cup \sigma\left[V_{2 m+1}\right] .
$$

Then use $\sigma$ to define neighborhoods of the points $\lambda_{\alpha}+2 n+1$ :

$$
B\left(\lambda_{\alpha}+2 n+1, k\right)=\sigma\left[B\left(\lambda_{\alpha}+2 n, k\right)\right] \text {. }
$$

$X \times X$ normal. We now construct $X$ so that $X \times X$ is a normal space. From Lemma 1, we need to construct $X$ so that all wispy subsets of $X \times X$ meet $\Delta$.

Suppose that $X$ is an Ostaszewski space. Then $X \times X$ is locally countable, so compact sets are countable. Thus, an easy closing-off argument gives: if $A \subseteq X \times X$ is wispy then there is a club $C \subseteq \omega_{1}$ such that if $\alpha \in C$, then $A \cap(\alpha \times \alpha)$ is wispy in $X_{\alpha} \times X_{\alpha}$. Thus, a diamond sequence can be used to guess potential wispy sets as we build $X$.

We use a diamond sequence of the form $\left\{E_{\alpha}: \alpha \in \omega_{1}\right\}$, where $E_{\alpha} \subseteq \lambda_{\alpha} \times \lambda_{\alpha}$ and for each $E \subseteq \omega_{1} \times \omega_{1}$ there is an $\alpha \in \omega_{1}$ such that $E_{\alpha}=E \cap\left(\lambda_{\alpha} \times \lambda_{\alpha}\right)$. (Recall that on a club, $\lambda_{\alpha}=\alpha$.)

We follow the basic construction, except when $\alpha=\nu+1$ and $E=E_{\nu} \backslash \Delta$ is large in $X_{\nu} \times X_{\nu}$. Let $A$ and $B$ be the closed discrete sets from the basic construction. Select distinct points $a_{0}, b_{0}, c_{0}$, and $d_{0}$, with $\left(c_{0}, d_{0}\right) \in E$, $a_{0} \in A$, and $b_{0} \in B$. Select pairwise disjoint, clopen-compact neighborhoods $U_{0}, V_{0}, R_{0}$, and $S_{0}$ of $a_{0}, b_{0}, c_{0}$, and $d_{0}$, respectively, of diameter at most 1. Since $E$ is large, there are additional distinct points $a_{1}, b_{1}, c_{1}$, and $d_{1}$, with $\left(c_{1}, d_{1}\right) \in E, a_{1} \in A$, and $b_{1} \in B$. Choose neighborhoods of these points as before, this time with diameter at most $1 / 2$, so that the family of all neighborhoods chosen so far is discrete.

Continuing by induction, we obtain a discrete family of neighborhoods, to which we apply the proof of Lemma 0 . This gives us the space $X_{\alpha}$ and completes the successor step of the induction.

Note that if $E=E_{\nu} \backslash \Delta$ is large in $X_{\nu} \times X_{\nu}$, then by induction, $\operatorname{cl}_{X \times X} E \supseteq$ $\left(\nu+1, \omega_{1}\right) \times\left(\nu+1, \omega_{1}\right)$. Since each uncountable subset is guessed on a stationary set and wispy reflects on a club, this gives that each wispy set meets the diagonal, and so $X \times X$ is normal.

$\beta X \times Y$ and $X \times \beta Y$ normal. This construction is similar to the previous one, except that we are building $X$ and $Y$ simultaneously, and at stage $\alpha=\nu+1$ we ask if the entire set $E_{\alpha}$ is large in $X_{\nu} \times Y_{\nu}$. We leave the details to the interested reader. 


\section{REFERENCES}

[B] A. Bešlagić, A Dowker product, Trans. Amer. Math. Soc. 292 (1985), 519-530.

[D] K. Devlin, Variations on diamond, J. Symbolic Logic 44 (1979), 51-58.

[F] W. G. Fleissner, Forcing Ostaszewski spaces (to appear).

[FKL] W. G. Fleissner, J. Kuleza, and R. Levy, Cofinality in normal almost compact spaces, Proc. Amer. Math. Soc. 113 (1991), 503-511.

[K] K. Kunen, Set theory, North-Holland, Amsterdam, 1980.

[O] A. Ostaszewski, On countably compact, perfectly normal spaces, J. London Math. Soc. 14 (1976), 505-516.

[P] T. Przymusinski, Products of normal spaces, Handbook of Set-theoretic Topology (Kunen and Vaughn, eds.), North-Holland, Amsterdam, 1984, pp. 781-826.

Department of Mathematics, Kansas University, Lawrence, Kansas 66045 E-mail address: LABERGE@UKANVAX.BITNET, LABERGE@KUHUB.CC.UKANS.EDU 